Annals of the Rheumatic Diseases 1981, 40, 615-627

\title{
Heberden Society: Combined Meeting, September 1981
}

A combined meeting of the Heberden Society, the British Association for Rheumatology and Rehabilitation, the Royal Society of Medicine Section of Rheumatology and Rehabilitation, and the Irish Society of Rheumatology and Rehabilitation was held at Newcastle upon Tyne, on 17-18 September 1981. The chairman of the meeting was Dr B. E. W. Mace. The following papers were presented.

The interactions of crystals and polymorphonuclear leucocytes monitored by luminol dependent chemiluminescence. P. N. Platt, S. Jeffery, L. Wilson, I. D. Griffith. J. H. Burn Laborat ory, University Department of Rheumatology, University of Newcastle upon Tyne.

In recent years the crystal-induced diseases have become established as a distinct group of conditions linked by a common pathogenesis. The interactions between polymorphs and crystals is an important factor in the production of these diseases.

When polymorphs phagocytose bacteria or yeasts, light is released as a chemiluminescence reaction related to the production of superoxide radicals. This reaction can be amplified chemically by the use of the chemical luminol. We have taken this technique and applied it to the study of polymorphonuclear leucocytes and crystals.

The production of chemiluminescence appears to correlate with the inflammatory potential of crystal preparations as measured by animal models. This is well illustrated by the chemiluminescence reaction produced by amorphous and microcrystalline sodium urate. Amorphous sodium urate is noninflammatory in animal models and produces only a weak reaction, less than $10 \%$ of the response to the intensely inflammatory microcrystalline sodium urate.

Crystal surfaces avidly bind protein, which has been suggested as being of importance in the modification of inflammatory potential. The chemiluminescence reaction of uncoated microcrystalline sodium urate was $64 \pm 3.5$ units, the response to Cohn fraction II coated urate was significantly enhanced at $130 \pm 7 \cdot 1$ units, whereas the response to albumin-coated urate was at background levels $5 \pm 0 \cdot 3$ units.

Luminol-dependent chemiluminescence appears to be a useful method for the study of interaction of polymorphs and crystals which has an acceptable level of reproducibility (SEM $\pm 5.5 \%$ ) for 50 readings from 6 batches of polymorphs.

Ferritin: a role in the pathogenesis of inflammatory joint disease. D. R. Blake, A. Potter, J. Price, N. D. Hall, P. A. Bacon, J. M. C. Gutteridge,* B. Halliwell. $\dagger$ RNHRD, Bath; *NIBSC, London; $\dagger$ King's College, London.

Oxygen-free radical intenuediates may exacerbate rheumatoid inflammation. The most toxic of these, the hydroxyl radical, degrades hyaluronic acid and promotes lipid peroxidation. Its production is stimulated by iron but inhibited by catalase. The trapping of iron in apoferritin as ferritin is therefore considered advantageous.

However, we have found that (1) concentrations of ferritin comparable to those found in the synovial fluid of patients with active rheumatoid synovitis can inhibit catalase, and (2) the rate of lipid peroxidation of bovine phospholipid liposomes is increased sixfold by ferritin. Ferritin production from apoferritin may therefore be harmful.

Further support for this concept has been gained from the histological study of 150 synovial biopsies. Apoferritin was detected by an immunoperoxidase technique and ferric iron (free and in haemosiderin) by ferrocyanide stain. 
Ferric iron was present in both inflammatory and noninflammatory states. Apoferritin was present only in inflammatory conditions. Most apoferritin was found in rheumatoid synovitis. When apoferritin existed in close proximity to its degradation product haemosiderin (suggesting the apoferritin was iron saturated) the inflammatory disease was severe. Systemic RA patients had most apoferritin/ haemosiderin, while early RA patients without apoferritin, or with apoferritin but no haemosiderin, had a mild course.

The data suggest that ferritin iron may promote inflammation, and we are exploring the therapeutic effect of its removal.

The importance of clinical sacroiliac tests in the diagnosis of early ankylosing spondylitis. P. W. Blower, A. J. Griffin. Greenwich District Hospital.

Four tests for sacroiliac pain were compared independently by 2 observers in 33 people with ankylosing spondylitis (AS) and 33 with mechanical low back pain (MLBP). All tests showed satisfactory interobserver reproducibility $(p=0.001$ for all), but only 2 , forcing downwards over the anterior superior iliac spines and forcing over the lower half of the sacrum, showed significant specificity for AS ( $\mathrm{p}=0.05$ and 0.01 ).

These 2 tests were applied in a prospective study by one observer to 500 Caucasians between the ages of 16 and 65 who presented consecutively to an outpatient clinic with a main complaint of low back pain \pm leg pain. Positive tests were associated with AS, HLA B27 in the presence of normal radiographs and pregnancy/puerperium $(p=0 \cdot 001,0 \cdot 001,0 \cdot 001)$.

Of 4 patients with positive tests, HLA B27, and normal radiographs, only one had limitation of all lumbar movements. All had chest expansions greater than $2.5 \mathrm{~cm}$ and normal ESRs. It is suggested that these patients had early AS.

Of 20 patients with definite AS 14 had limitation of all lumbar movements, 8 had positive sacroiliac tests, and 6 had a chest expansion of $2.5 \mathrm{~cm}$ or less.

Radioisotope studies of painful shoulder syndromes. T. J. Daymond, E. D. Williams, W. C. Dick. Department of Rheumatology, District General Hospital, Kayll Road, Sunderland.

The aetiology of shoulder lesions remains obscure; both extra-articular soft tissue inflammation and synovitis have been implicated. We have therefore studied patients suffering from painful shoulders using objective radioisotope methods to check any
Table 1 Results of radioisotope studies

\begin{tabular}{|c|c|c|c|c|}
\hline Disease & Patient & $\begin{array}{l}{ }_{\min }^{133} X e^{\mathrm{T}} / 2 \\
\end{array}$ & $\begin{array}{l}\text { Tc index } \\
\% \text { dose }\end{array}$ & $\begin{array}{l}\text { MDP index, } \\
\% \text { dose }\end{array}$ \\
\hline $\begin{array}{l}\text { Frozen } \\
\text { shoulder }\end{array}$ & $\begin{array}{r}1 \\
2 \\
3 \\
4 \\
5 \\
6 \\
7 \\
8 \\
9 \\
10 \\
11 \\
12\end{array}$ & $\begin{array}{r}25 \\
28 \\
17 \\
17 \\
15 \\
37 \\
24 \\
22 \\
29 \\
14 \\
153 \\
207 \\
22 \cdot 8 \\
7 \cdot 3\end{array}$ & $\begin{array}{l}0 \cdot 77 \\
1 \cdot 14 \\
1 \cdot 00 \\
1 \cdot 02 \\
0 \cdot 83 \\
0 \cdot 76 \\
1 \cdot 03 \\
0 \cdot 93 \\
0 \cdot 77 \\
0 \cdot 69 \\
0 \cdot 99 \\
0 \cdot 72 \\
0 \cdot 85 \\
0 \cdot 15\end{array}$ & $\begin{array}{l}0.41 \\
0.61 \\
- \\
1.05 \\
0.56 \\
0.59 \\
0.93 \\
0.78 \\
0.50 \\
0.66 \\
1.00 \\
0.58 \\
0.70 \\
0.21\end{array}$ \\
\hline $\begin{array}{l}\text { Rheuma- } \\
\text { toid } \\
\text { arthritis }\end{array}$ & $\begin{array}{l}1 \\
2 \\
3 \\
4 \\
5\end{array}$ & $\begin{array}{c}6 \\
14 \\
22 \\
25 \\
21 \\
17 \cdot 6 \\
7 \cdot 64\end{array}$ & $\begin{array}{l}0.75 \\
0.70 \\
0.80 \\
1 \cdot 18 \\
1.25 \\
0.94 \\
0.26\end{array}$ & $\begin{array}{l}- \\
0.48 \\
0.51 \\
1.43 \\
0.71 \\
0.78 \\
0.44\end{array}$ \\
\hline $\begin{array}{c} \\
\text { Mean } \\
\text { SD }\end{array}$ & $\begin{array}{l}1 \\
2 \\
3 \\
4 \\
5 \\
6 \\
7 \\
8 \\
9\end{array}$ & $\begin{array}{c}16 \\
60 \\
24 \\
17 \\
32 \\
100 \\
285 \\
120 \\
49 \\
33 \cdot 0 \\
17 \cdot 96\end{array}$ & $\begin{array}{l}0.58 \\
0.95 \\
0.98 \\
1.46 \\
0.57 \\
0.71 \\
1.01 \\
0.91 \\
0.95 \\
0.90 \\
0.27\end{array}$ & $\begin{array}{l}0.57 \\
0.48 \\
0.57 \\
0.67 \\
0.50 \\
0.81 \\
0.86 \\
0.66 \\
0.88 \\
0.67 \\
0.15\end{array}$ \\
\hline
\end{tabular}

association between their characteristics and those of patients suffering from shoulder synovitis due to rheumatoid arthritis.

Twelve patients with frozen shoulders and 9 with painful arc syndrome were studied and compared with 5 patients suffering from rheumatoid arthritis. Clinical assessment excluded alternative diagnosis of painful shoulder. The clearance rate of xenon-133 from the shoulder after intra-articular injection and uptake indices for Tc-99m pertechnetate and Tc-99 methylene diphosphonate were measured consecutively.

The results are shown in Table 1 . Values for ${ }^{133} \mathrm{Xe} \mathrm{T} / 2$ min over 100 are excluded from the mean. The interpretation and statistical analysis of these results will be discussed.

Pyopericardium associated with rheumatoid disease. M. Martin, P. Walker, V. Wright, P. Dieppe. Department of Rheumatology, Bristol Royal Infirmary, and Rheumatism Research Unit, University of Leeds.

Pericarditis in association with rheumatoid disease is usually asymptomatic but occasionally progresses 
to acute tamponade, pericardial constriction, or a combination of these 2 conditions. Each of these severe complications is potentially fatal, and several recent reviews have recommended early pericardectomy.

Infection developing within an aseptic pericarditis is now well recognised in other debilitating chronic diseases, in particular uraemia, malignancy, or following thoracic surgery. ${ }^{1}$ There have been only 2 single case reports of this condition in patients with rheumatoid arthritis. We have reviewed 4 further cases, and in $\mathbf{3}$ an infecting organism was isolated.

The clinical features suggest that a pyopericardium occurs at any stage during the course of a patient's arthritis. In each case, at presentation, atypical signs of constriction were found and in no patient was an infected pericardium suspected. None of the patients were febrile, and 3 died as a result of the disease.

This rare complication is difficult to diagnose and requires the clinician to maintain a high index of suspicion in the appropriate clinical setting. Early diagnosis should be attempted and pericardectomy is recommended.

\section{Reference}

1 Klacsman P G, Bulkley B H. Hutchins G M. Am J Med 1977; 64: 666-73.

Chondromalacia patellae: a clinical and arthroscopic study. I. D. Griffiths, I. M. Pinder. Departments of Rheumatology and Orthopaedics, Freeman Hospital, Newcastle upon Tyne.

Painful, creaking knees are a common cause of referral to rheumatology units and in the absence of radiological changes frequently labelled as chondromalacia patellae (CMP). This study is part of a prospective analysis of aetiological and clinical features and their correlation with arthroscopic findings in patients with suspected CMP.

A total of 102 patients had arthroscopies for suspected CMP, the clinical criteria being knee pain, associated with retropatellar crepitus, and pain on patello-femoral rubbing. Sixty-eight of these patients had CMP confirmed at arthroscopy. A further 5 patients also had CMP at arthroscopy, but in this group the clinical diagnosis had been meniscal damage. Of this total group of 73 patients, 10 had bilateral signs and symptoms. A variety of aetiological factors appeared to be relevant, including previous fractured patella (4), minor trauma (29), previous menisectomies (12), hypermobility (12), in this later group the disease frequently being bilateral.

In the group of 34 patients with clinical evidence of CMP but without arthroscopic confirmation 10 had normal arthroscopic appearances, 18 had synovial hyperaemia with or without proliferation, and the remainder had either early degenerative changes, plical folds, or adhesions.

Therefore the clinical diagnosis of CMP is arthroscopically confirmed in only 2 out of 3 patients, and a variety of factors may be implicated in this disorder.

Rheumatological problems in Gaucher's disease. P. Beighton, ${ }^{*}$ J. Goldblatt, S. Sacks. $\dagger{ }^{*}$ Department of Human Genetics, Medical School, University of Cape Town, Observatory 7925, Cape, South Africa; $\dagger$ Department of Orthopaedic Surgery, University of the Witwatersrand, Johannesburg, South Africa.

Rheumatological problems are an important feature of the adult, chronic or nonneuropathic form of Gaucher's disease. Thirty-five patients have been investigated and, of these, 29 had significant skeletal complications. Twenty-one had nonspecific bone pains, 14 had episodes of pseudo-osteomyelitis, and 2 had acute haematogenous osteomyelitis. Twenty experienced collapse of femoral heads, and in 9 prosthetic joint replacement had been successfully undertaken. Four had kyphoscoliosis due to wedging of vertebral bodies, and 3 had pathological fractures of tubular bones.

Twenty-eight of the patients were of Ashkenazi Jewish stock, and the minimum prevalence of the disorder in this group in South Africa is 1 in 4300 . If skeletal problems and splenomegaly coexist in a Jewish patient, the diagnosis of Gaucher's disease warrants serious consideration.

Platelets and rheumatoid arthritis. M. Farr, D. L. Scott, T. Constable, R. Hawker, C. F. Hawkins. Queen Elizabeth Hospital, Birmingham.

It is common clinical practice to monitor platelet counts in rheumatoid arthritis (RA), and we have evaluated their relationship to disease activity. A cross-sectional study (130 patients with RA) showed platelet counts were commonly high $\left(>400 \times 10^{9} \mathrm{~L}\right)$ and correlated with parameters such as the articular index, albumin, and rheumatoid factor (SCAT 
titre). A second cross-sectional study (100 patients with RA) showed platelet counts were closely related to the ESR and its 'equivalents' (such as the ZSR, plasma viscosity, C-reactive protein, orosomucoid, and others). A longitudinal study (15 patients with RA followed over 4 years) showed a close relationship between platelet counts and exacerbations and improvements in the disease. However, such changes are not specific for RA; in a longitudinal study of 15 patients with Crohn's disease (over 4 years) platelet counts also followed disease activity and the ESR.

Studies with ${ }^{111}$ indium-labelled platelets showed a significant decrease in platelet survival in active RA and evidence of deposition of platelets around inflamed joints, but there was little evidence of abnormal platelet aggregation.

Although platelet counts are usually monitored to detect adverse reactions to therapy, they can also provide the clinician with significant additional information of disease activity.

Sideroblastic Anaemia presenting as arthritis secondary to iron overload. J. R. Kirwan, M. A. Fitzcharles, B. T. Colvin, ${ }^{*}$ H. L. F. Currey. Bone and Joint Research Unit and *Department of Haematology, The London Hospital, London E1 1 BB.

Arthritis is a well recognised feature of idiopathic haemochromatosis and has been reported after repeated blood transfusions and in hereditary spherocytosis. It is thought to result from the iron overload associated with these latter conditions. ${ }^{1}$

We report 2 cases of sideroblastic anaemia with iron overload whose presenting symptom was an arthritis of small and large joints. One patient had marked spinal symptoms which were severe enough to suggest ankylosing spondylitis and the pattern of arthritis in the other resembled seronegative rheumatoid arthritis. The progression of arthritis in the second patient was accompanied by increasing sideroblastic changes in serial bone marrow aspirates.

We believe these to be the first cases described of an arthropathy associated with iron overload secondary to sideroblastic anaemia.

\section{Reference}

${ }^{1}$ Schumacher H R. Arthritis Rheum 1964; 7: 41-50.
Biomechanics of the patello-femoral articulation with special reference to the influence of varous deformity, and its correction. K. Fujikawa, B. B. Seedhom, V. Wright. Leeds.

The knee joint is often regarded as 2 independent compartments, and surgical treatment for arthritis is carried out on either compartment not always bearing in mind the consequences of the surgery on the other. This study investigates the effect of correction of varus deformity, by high tibial osteotomy and introduction of a bony wedge, on the contact and congruence of the patellofemoral (p-f) joint in necropsy knees. The patterns of contact in the p-f compartment was established for angles of flexion of $25,45,60,90$, and $115^{\circ}$. These were repeated on each specimen after imposing a simulated varus deformity (by high tibial osteotomy and the introduction of a bony wedge) of 5,10 , and $15^{\circ}$. Up to $5^{\circ}$ varus deformity, the p-f articulation was minimally influenced. At more than $10^{\circ}$ varus deformity, the $p$-f congruity was definitely destroyed by marked changes in the contact pattern. Mainly the contact areas on the patella concentrated on the medial part of the medial facet proper. The investigation showed that hyperstresses should occur in the medial compartment. This was contrary to the clinical finding that the patella is normally displaced laterally and the lateral facet hyperstressed so that the majority of the p-f OA is dominant in the lateral compartment. This indicates that the abnormal lateral torsion of the upper part of the tibia, which is normally present with the varus deformity (from a previous study on $3000 \mathrm{OA}$ knees), is responsible for this change in the site of the OA lesions.

This study demonstrates that the sudden imposition of a varus deformity can destroy the congruence of the joint. It is also proposed that the sudden correction of the varus deformity in a knee, whose surfaces have been remodelled, can equally destroy the congruence of the joint and cause high stresses to occur in the p-f compartment.

Serological features of morphea. P. N. Platt, W. Carson Dick, P. Friedman, M. Hazel. Departments of Rheumatology and Dermatology, University of Newcastle upon Tyne.

Scleroderma is a characteristic skin appearance which is common to the conditions of morphea, linear scleroderma (LS) and progressive systemic sclerosis (PSS). The aetiology of these conditions is unknown. However, there is some evidence to suggest that immunological mechanisms play a role in progressive systemic sclerosis. The presence of 
hypergammaglobulinaemia, antinuclear antibodies, rheumatoid factor, lymphocytic infiltrates, vascular deposition of immunoglobulins and complements, and antibodies to RNA have been described in PSS.

Despite similar early histological and clinical features of the involved skin in these 3 conditions the evidence for abnormal immunological functions in morphea and linear scleroderma is fragmentary. We have studied patients with morphea and linear scleroderma for the presence of these abnormalities

Table 2

\begin{tabular}{llll}
\hline & Morphea & $L S$ & $P S S$ \\
\hline C1q & $5 / 21(23 \%)$ & $2 / 6(33 \%)$ & $5 / 8(62 \cdot 5 \%)$ \\
ENA & $0 / 21$ & $0 / 6(16 \%)$ & $3 / 8(37 \cdot 5 \%)$ \\
ANA & $0 / 21$ & $1 / 6(16 \%)$ & $3 / 8(37 \cdot 5 \%)$ \\
DNA binding & $0 / 21$ & $0 / 6$ & $1 / 8(12 \cdot 5 \%)$ \\
Rheumatoid factor & $0 / 21$ & $1 / 6(16 \%)$ & $0 / 8$ \\
Autoantibodies & $6 / 35(17 \%)$ & $0 / 6$ & $2 / 8(25 \%)$ \\
\hline
\end{tabular}

Immune complex levels are elevated in a proportion of patients with all 3 conditions, the highest proportion being in those with PSS. The other significant abnormality was the presence of a group of male patients with antithyroid antibodies amongst those with morphea ( 3 out of 12 patients $p=0.001$ ). Immunological abnormalities and autoantibodies do occur in localised scleroderma but in a smaller proportion than in PSS.

Gold distribution in blood following administration of Myocrisin and auranofin. D. Lewis, A. I. A. Rogers, E. Smith, H. A. Capell. Centre for Rheumatic Diseases, University Department of Medicine, Glasgow Royal Infirmary, and Department of Pure and Applied Chemistry, Strathclyde University, Glasgow.

Triethylphosphine gold (auranofin) has been shown in animal studies to produce a lower tissue accumulation and a different plasma/cell gold ratio from that found with sodium aurothiomalate (Myocrisin).

In the course of a clinical study comparing conventional doses of Myocrisin and auranofin $6 \mathrm{mg}$ daily we have measured gold levels in whole blood, in plasma, and in a lysate from red cells in 32 patients (16 in each group). Blood levels increase with time with both drugs but whole blood and plasma gold is higher in the Myocrisin group. Red cell lysate levels are higher in the patients receiving auranofin and there is a significant difference in the lysate/plasma ratio in the 2 groups (Table 3 ).

Within the Myocrisin group there were markedly higher lysate gold concentrations in the smokers compared with nonsmokers, but this difference was not observed in patients on auranofin (Table 3).
Table 3 Concentrations of gold after 12 weeks of therapy

\begin{tabular}{|c|c|c|}
\hline & $\begin{array}{l}\text { myocrisin } \\
n=16\end{array}$ & $\begin{array}{l}\text { auranofin } \\
n=16\end{array}$ \\
\hline 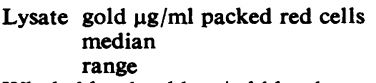 & $\begin{array}{l}0 \cdot 25 \\
(0 \cdot 0-1 \cdot 5)\end{array}$ & $\begin{array}{l}0.9 \\
(0.4-1 \cdot 9)\end{array}$ \\
\hline $\begin{array}{l}\text { Whole blood gold } \mu \mathrm{g} / \mathrm{ml} \text { blood } \\
\text { median } \\
\text { range }\end{array}$ & $\begin{array}{l}2 \cdot 45 \\
(1 \cdot 0-3 \cdot 3)\end{array}$ & $\begin{array}{l}0 \cdot 8 \\
(0 \cdot 3-1 \cdot 6)\end{array}$ \\
\hline $\begin{array}{l}\text { Plasma gold } \mu \mathrm{g} / \mathrm{ml} \text { serum } \\
\text { median } \\
\text { range }\end{array}$ & $\begin{array}{l}3 \cdot 5 \\
(1 \cdot 8-4 \cdot 8)\end{array}$ & $\begin{array}{l}1 \cdot 0 \\
(0 \cdot 4-2 \cdot 6)\end{array}$ \\
\hline $\begin{array}{l}\text { Lysate: Plasma (all patients) } \\
\text { median } \\
\text { range }\end{array}$ & $\begin{array}{l}0 \cdot 13 \\
(0-0 \cdot 61)\end{array}$ & $\begin{array}{l}0.9 \\
(0 \cdot 35-2 \cdot 7)\end{array}$ \\
\hline $\begin{array}{l}\text { Lysate: plasma (smokers) } \\
\text { median } \\
\text { range }\end{array}$ & $\begin{array}{l}0.26 \\
(0.15-0.61)\end{array}$ & $\begin{array}{l}0.9 \\
(0.59-1.5)\end{array}$ \\
\hline $\begin{array}{l}\text { Lysate: plasma (nonsmokers) } \\
\text { median } \\
\text { range }\end{array}$ & $\begin{array}{l}0.056 \\
(0-0.24)\end{array}$ & $\begin{array}{l}0.9 \\
(0 \cdot 35-2 \cdot 7)\end{array}$ \\
\hline
\end{tabular}

The change in concentration of haemoglobin between 0 and 12 weeks was similar in the Myocrisin and auranofin treated patients and in a concomitant group of 17 patients receiving auranofin placebo.

After 6 months of Myocrisin therapy the gold levels in those on maintenance injections (3 or 4 weekly) fell in plasma and whole blood, and almost disappeared in the red cell lysate. In contrast the levels in the auranofin group (who continued $6 \mathrm{mg}$ daily) tended to remain constant or to increase.

The relevance of the observed differences in gold distribution between the 2 compounds remains uncertain.

The relationship between quadriceps size and strength in normal young adults. A. Young, M. Stokes, I. C. R. Walker, ${ }^{*}$ D. Newham.* Oxford Rehabilitation Research Unit and *University College, London School of Medicine.

The linear relationship between quadriceps strength and the body weight of normal subjects is a valuable aid to the objective evaluation of the complaint of weakness. ${ }^{1}$ Nevertheless the contribution of atrophy to the weakness has only been judged subjectively or by extrapolation from measurements of muscle fibre size. Now, however, it is possible to measure quadriceps cross-sectional area (CSA) by ultrasonography. ${ }^{2}$ Is the relationship between the strength and the CSA of the normal quadriceps sufficiently close to be applicable in clinical practice?

The relationship between body weight, quadriceps CSA and quadriceps strength was studied in 25 normal subjects (11 male, 14 female) aged 19-48 (median age 25).

As expected, there was a weak correlation 
$(r=0.54)$ between the maximum voluntary isometric torque exerted by the stronger quadriceps and body weight, with men tending to be stronger for their weight than women. The sex difference disappears and the correlation is much closer $(r=0.84)$ when the torque exerted by the stronger quadriceps is compared with its CSA. The strength of this relationship suggests that it could be used to indicate how much of a patient's weakness is due to atrophy.

\section{References}

1 Edwards R H T, Young A, Hosking G P, Jones D A. Clin Sci Molec Med 1977; 52: 283-90.

2 Young A, Hughes I, Russell P, Parker M J, Nichols P J R. Rheumatol Rehabil 1980; 19: 141-8.

Effect of klebsiella capsular antisera on lymphocytes from patients with ankylosing spondylitis. R. Shinebaum, E. M. Cooke, J. Siegersletter, V. Wright. Leeds.

It has been suggested that klebsiella is involved in the pathogenesis of ankylosing spondylitis (AS), a disease closely linked to the possession of HLA B27, and that a possible mechanism for such involvement is molecular mimicry. Some studies have linked exacerbations of AS to intestinal carriage of klebsiella, and partial cross-reactivity between klebsiella antigen(s) and B27 lymphocytes has been shown in vitro.

In this study lytotoxicity of 52 klebsiella capsular antisera for lymphocytes from ten B27-positive patients with AS and from 8 B27-negative normal controls was examined. None of the antisera were lytotoxic for lymphocytes from 9 patients, but several antisera were lytotoxic for lymphocytes from 1 patient. There were no signicant differences between the results obtained from the patients and controls.

We were generally unable to demonstrate crossreactivity between klebsiella and B27 lymphocytes in this system. If klebsiella is important in the pathogenesis of AS, the results of this investigation suggest that it is unlikely that the capsular antigen is involved.

Radioisotopic scanning of sacroiliac joints in the diagnosis of early scanning. I. D. Griffiths, B. Clayton, T. J. Daymond, M. Thompson. Department of Rheumatology, Royal Victoria Infirmary, Newcastle upon Tyne NE1 4LP.

The early diagnosis of ankylosing spondylitis in the absence of radiological changes may present difficulties. This study is based upon a prospective clinical and radio clinical and radiological assessment of young adults presenting with back pain in whom ${ }^{99}$ technetium methylene diphosphonate quantitative sacroiliac scans have been performed. Ratios of isotope uptake in mid-portion of the sacroiliac joint and the corresponding area of the sacrum have been taken. Thirty-six patients with low back pain, morning stiffness, and possessing HLA B27 MHC antigen have been documented. Pelvic radiographs \& were graded for sacroiliitis on a $0-4$ scale by $2 \overrightarrow{0}$ independent observers. Of the group with $0 / 1$ grade $14 / 15$ had increased isotope uptake in at least one $\vec{\omega}$ sacroiliac joint (mean ratio 1.7). For the other grades the corresponding figures were: grade II $7 / 7$ ? $(2 \cdot 45)$, grade III $7 / 10(1 \cdot 59)$, grade IV $0 / 4(1 \cdot 08)$. A group of 8 patients, 2 of whom had unequivocal radiological sacroiliitis, were seen in whom low back pain and morning stiffness were marked; all were B27-negative but 5 were HLA B7 Creg + and all had increased scan uptake. Sequential data suggested that increased isotope uptake persists for months and possibly identifies a discrete subgroup of back pain sufferers. These data support the view that quantitative sacroiliac scanning is a useful addition in the investigation of low back pain.

Subnormal binding of dexamethasone by poly morphonuclear leucocytes (PMNLs) in rheumatoid and other arthritis. I. P. Braidman, K. Livingstone, I. W. Tomlinson, M. I. V. Jayson, M. K. Jasani. Rheumatic Diseases Centre, University of Manchester, Hope Hospital, Salford; Ciba-Geigy Research Centre, Horsham, West Sussex.

In the rat physiological circulating levels of corticosterone exert a detectable anti-inflammatory (AIF) action, ${ }^{1}$ by interactions with high-affinity cytoplasmic receptors. ${ }^{2} 3$ Hypothalmo-pituitaryadrenal function in RA patients who have not been treated with AIF steroids is comparable with that of nondiseased controls. ${ }^{4}$ So the question is why does joint inflammation in RA, once established, persist in many patients?

Previously we have shown that rheumatoid synovial tissue cells exhibit high affinity binding for dexamethasone $\left(\mathrm{K}_{\mathbf{d}} 10 \mathrm{nM}\right)$ but PMNLs from RA synovial fluid do not $\left(K_{d} 15-250 \mathrm{nM}\right){ }^{5}$ We have therefore compared dexamethasone binding characteristics of PMNLs from venous blood from various types of arthritis and found them to be normal in all 5 osteoarthritis patients studied but subnormal in 11 with RA and 9 with seronegative polyarthritis. In further experiments incubation of blood PMMLs from controls at $37^{\circ} \mathrm{C}$ for $30 \mathrm{~min}$ in plasma from RA patients but not in control plasma 
Table $4 \quad K_{\mathrm{d}}\left({ }^{3} \mathrm{H}\right)$-dexamethasone $\left(\times 10^{-9} \mathrm{M}\right)$ of non-arthritic PMNLs postincubation at $37^{\circ} \mathrm{C}$ for $15 \mathrm{~min}$

\begin{tabular}{ll}
\hline Nonarthritic plasma & RA plasma \\
\hline 14.0 & 73.3 \\
9.2 & 54.9 \\
13.3 & 24.4 \\
12.0 & 23.5 \\
11.3 & 46.0 \\
13.4 & 55.0 \\
10.5 & 78.0 \\
\hline
\end{tabular}

was found to result in loss of high affinity binding (Table 4). These results suggest that in inflammatory arthritis there is a circulating factor which interferes with dexamethasone binding, and by implication makes PMNLs less responsive to endogenous cortisol. This could in part explain why joint inflammation persists in RA.

\section{References}

1 Sendelbeck L R, Yates F E. Am J Physiol 1970; 219 : 845-53.

2 Tsurufuji S, Kazuo S. Eur J Rheumatol 1978; 1 : 226-7.

3 Coote J, et al. Br J Pharmacol 1980; 70 : 101 P.

4 Jasani M K, et al. Q J Med 1968; 147: 407-21.

5 Braidman I P, et al. Agents Actions 1980; suppl 7: 233-6.

Rehabilitation of patients after myocardial infarction. M. A. Hossain, G. Sandler. H. E. Walker. District General Hospital, Barnsley.

There is divergence of opinion regarding the effect of physical exercise on patients after myocardial infarction (MI). Some workers consider that physical exercise plays an important role in the rehabilitation of patients with MI, but others consider that changes in mood and self confidence are more likely to be due to group therapy rather than exercise itself. We have carried out a progressive and supervised coronary rehabilitation programme (CRP) on a selected group of patients with MI to study its effect on physical fitness and on their return to work.

262 patients attended a 6-week course of CRP during the last $3 \frac{1}{2}$ years. The CRP started on the 2nd day of admission to the Coronary Care Unit $(\mathrm{CCU})$ and continued daily until discharge. Patients then attended a weekly session as an out-patient for 6 weeks. At the end of the CRP the patients were assessed by a 'coronary rehabilitation team' to consider whether the patient was fit to go back to work, or to consider retraining at the Employment Rehabilitation Centre (ERC).

$182(77 \%)$ of patients out of 237 who were employed before MI were able to go back to work within 10 weeks of MI, as opposed to 16 weeks when no active CRP was used. Thirty-seven patients could not complete the CRP due to recurrent angina, 15 patients died due to further MI. In conclusion we believe that physical exercise plays an important part in the rehabilitation of patients with MI. It not only helps to regain self confidence and prevent cardiac neurosis, but also enhances physical fitness and early return to work.

Significance of $\mathbf{p H}$ in synovial fluid. M. Farr, M. J. Kendall, A. M. Bold, C. F. Hawkins. Queen Elizabeth Hospital, Birmingham.

Analysis of synovial fluid may help in making a diagnosis and in assessing severity of the disease process. Since inflammation gives rise to tissue acidosis, ${ }^{1}$ determination of synovial fluid hydrogen on concentration might prove a useful measure which has the advantage of being simple to carry out.

$\mathrm{pH}$ of synovial fluid from knee joints of 30 patients with rheumatoid arthritis (RA) and 13 with osteoarthrosis (OA) was measured by an acid base analyser $\left(\mathrm{ABL}_{2}\right.$ Acid-Base Laboratory) using a technique which prevented contact with air.

$\mathrm{pH}$ was lower in synovial fluid from rheumatoid patients than from OA patients. A lower $\mathrm{pH}$ was found in patients with a higher ESR, and was associated with higher synovial fluid levels of acute phase proteins, namely, C-reactive protein, haptoglobin, and orosomucoid, and also a higher polymorph count.

A more acid fluid $\mathrm{pH}$ appears therefore to be associated with a greater degree of inflammatory activity. It is of interest partly because it is an extremely easy 'marker' to measure and also because the $\mathrm{pH}$ difference between synovial fluid and blood may influence the transfer of drugs into the joint, since lipid solubility of the drug may be determined by the extent to which the drug is ionised.

\section{Reference \\ 1 Menkin V. Science 1966; 123: 527, 3196.}

A phenylbutazone dose-finding study. H. A. Bird, J. R. Lowe, P. A. Leatham, W. W. Downie, P. D. Fowler, V. Wright. Clinical Pharmacology Unit, Royal Bath Hospital, Harrogate.

Phenylbutazone (PBZ) is more effective than placebo in the treatment of rheumatoid arthritis ${ }^{1}$ but efficacy is associated with side effects. The drug has a long half life, and it is likely that in routine clinical practice too high a dose is used and for too short a time. We have therefore established the 
minimum effective dose for each patient in a pharmacokinetic study aimed to separate responders from nonresponders and to establish whether a relationship exists between blood levels and side effects.

Thirty-two patients randomised between 3 groups were treated for 9 weeks. Each patient took 3 increasing dose levels of PBZ for periods of 3 weeks each dose. If response occurred at a low dose, patients did not proceed to higher doses. Dose levels were $90 \mathrm{mg}$, $150 \mathrm{mg}, 180 \mathrm{mg}, 240 \mathrm{mg}, 270 \mathrm{mg}, 300 \mathrm{mg}, 360 \mathrm{mg}$, and $450 \mathrm{mg} /$ day. The study was double blind. Paracetamol provided rescue analgesia. A definition of response was derived from change in 6 clinical assessments.

The maximum efficacy of PBZ in these patients with RA occurred at $270 \mathrm{mg} /$ day. Doses below this do not produce full benefit; no further improvement occurs at higher doses. There was no apparent difference between the plasma pharmacokinetic patterns for PBZ or oxyphenbutazone in 'responders' and 'nonresponders'. Although $7 / 32$ patients experienced side effects, there was no relationship between blood levels of PBZ or oxyphenbutazone and side effects.

\footnotetext{
Reference

1 Fjellström K E, et al. Acta Med Scand 1957; 157: suppl $3201-49$.
}

A pharmacological and clinical comparison of prednisolone and betamethasone in rheumatoid arthritis. M. E. Pickup, H. A. Bird, R. Marwah, H. AlShakarchi, J. S. Dixon, J. R. Lowe. Clinical Pharmacology Unit, Royal Bath Hospital, Harrogate.

Oral corticosteroids continue to be used in the treatment of rheumatoid arthritis for their profound anti-inflammatory effect. Their rational place may be in the rapid induction of remission in patients who need to remain physically active, prior to the more gradual remission provided by the subsequent use of antirheumatoid drugs, but there is little information on the relative safety and efficacy of different preparations in chronic dosage in rheumatoid arthritis.

Twenty patients with RA entered a single blind crossover study, receiving either prednisolone 8 $\mathrm{mg}$ /day or betamethasone $1 \mathrm{mg} /$ day, each for 2-week periods. Seven clinical assessments were performed at the start and at the end of each treatment period. In addition to routine laboratory tests of disease activity a synacthen test (11-hydroxysteroid levels before and $30 \mathrm{~min}$ after $250 \mathrm{mg}$ tetracosactrin) was performed at the start and at the end of each treatment period as well as an endogenous 11-hydroxy- corticosteroid profile of samples at 0900,1200 , and $1700 \mathrm{~h}$.

Clinical improvement occurred in all patients. There was no significant difference between drugs at this dosage, though a slight patient preference for prednisolone emerged. However, both steroid profile and synacthen test showed more pronounced adrenal suppresion with betamethasone.

With comparable efficacy and less adrenal के suppression prednisolone appears the drug of choice $\overrightarrow{0}$ for chronic dosage. Moreover its formulation in $1 \mathrm{mg}$ tablet size allows easier reduction in dose.

Pharmacokinetic studies of tiaprofenic acid in patients suffering from rheumatoid arthritis. T. J. Daymond, R. Herbert. Department of $\dot{\sigma}$ Rheumatology, Sunderland and district hospital, $\vec{o}$ and Department of Pharmacy, Sunderland $\frac{9}{5}$ Polytechnic, Sunderland.

Nonsteroidal anti-inflammatory drugs are widely prescribed in rheumatological practice. The sequential timing of administration has been dependent on pharmacokinetic studies of blood levels, which may not reflect drug levels in other tissues. Pharmo acokinetic studies of tiaprofenic acid (Ta) were performed to obtain a profile of this drug in serure and synovial fluid.

Eleven patients suffering from rheumatoid arthritis were studied. Patients took $200 \mathrm{mg}$ Ta at $2200 \mathrm{~h}$ and fasted over night. $200 \mathrm{mg}$ Ta was given after fasting samples were taken and regular blood obtained during an $8 \mathrm{~h}$ period. Seven of these patients had knee effusions and synovial fluid was aspirated at regular intervals during the same $8 \mathrm{~h}$ period. Serum and synovial fluid levels of $\mathrm{Ta}$ were measured by HPLC.

Peak serum levels of Ta were found after 40$90 \mathrm{~min}$ (mean value $23.8 \mu \mathrm{g} / \mathrm{ml}$ ) with a mean half life of $1.5 \mathrm{~h}$. Synovial fluid levels of Ta remained relatively constant throughout the $8 \mathrm{~h}$ period value $6 \cdot 9 \mu \mathrm{g} / \mathrm{ml}$ ) and independent of serum levels.

These results confirm that $\mathrm{Ta}$ penetrates the synovial fluid membrane and is retained in the synovial fluid. They also suggest that drug regimens in rheumatoid arthritis should not necessarily be dependent on the serum half life.

Polymyalgia rheumatica (PMR) and antimitochondrial antibodies (AMA). M. A. Sattar, M. I. D. Cawley, J. C. Robertson. Department of Rheumatology, Southampton General Hospital.

Abnormal tests of liver function are well recognised in the clinical syndrome of PMR. The most com- 
monly noted abnormality is a raised serum alkaline phosphatase (ALP). PMR has a recognised association with giant cell arteritis and some other systemic diseases. A possible relationship between primary biliary cirrhosis (PBC) and PMR has recently attracted interest. We have therefore studied further the association between PMR, liver function, and AMA, the immunological marker of PBC. We reviewed the clinical records of 36 patients with well documented PMR with special reference to the following: (a) Biochemical liver function tests including serum bilirubin, aspartate transaminase (AST), alanine transaminase (ALT), ALP, 5nucleotidase (5NT), and serum proteins; $(b)$ circulating autoantibodies including AMA; and (c) needle liver biopsy.

In all cases bilirubin, AST, and ALT were normal. ALP was raised in 19 patients and 5NT in 10. Eleven had circulating AMA, of which 7 had titres over $1 / 64$. Only 3 liver biopsies were available, and all showed changes of mild nonspecific hepatitis. These observations confirm that some abnormalities of hepatic function are common in PMR, as in other inflammatory rheumatic diseases. The finding of AMA in nearly 1 in 3 of these patients suggests either a coincidental serological overlap between PMR and PBC or an aetiopathogenetic association between some cases of the 2 diseases.

Antibodies to type II collagen-comparison of immunological methods and clinical features in rheumatoid arthritis (RA). R. B. Clague, ${ }^{*}$ S. Firth, ${ }^{*}$ P. J. L. Holt, ${ }^{*}$ J. Skingle, C. L. Greenbury, M. Webley. Department of Rheumatism,* Stopford Building, Manchester, and Departments of Pathology and Rheumatism, Stoke Mandeville Hospital.

A collaborative study was carried out using 6 different assays for the detection of antibodies to type II collagen; passive haemagglutination using native type II collagen from human nucleus pulposus (HA) or bovine nasal cartilage (HB); IgG immunofluorescence on rat tracheal cartilage (IF): and IgG radioimmunoassays using either denatured (denat.
II) or native (nat. II) bovine type II collagen or cubes of bovine nasal cartilage (cart.). Each laboratory exchanged 50 coded sera with a high proportion of positive and controls (79 RA ( +5 duplicates), 10 normals, 1 osteoarthritis, and 5 non-RA) allowing analysis of 100 sera. The results and codes were exchanged by post simultaneously. The number of positive sera and correlations between the methods are shown in Table 5.

Retrospective analysis of the clinical features on the $\mathbf{4 2}$ patients with elevated levels (nat. II )and 34 RA patients with normal levels showed very few differences. Both groups were well matched for age, sex ratio, disease duration, ESR, SCAT and latex, previous joint surgery, and steroid therapy. More patients in the collagen antibody-positive group were receiving conventional second-line therapy $(44 \% \mathrm{v}$ $24 \%$ ) and had severe erosive changes ( $41 \%$ v $24 \%$ ), though patients with normal levels had a higher incidence of nodules (56\% v. $24 \%)$.

There were good correlations between antibody levels to type II collagen measured by different methods. The significance of these antibodies in RA remains uncertain, though they were associated with a higher incidence of erosive disease.

Uveitus and cross-reactivity studies between vitreous humour and klebsiella, using radioimmunoassay. A. Ebringer, H. Avakian, J. Welsh. Immunology Unit, Queen Elizabeth College, and Department of Rheumatology, Middlesex Hospital, London.

Uveitis occurs in some patients with ankylosing spondylitis, and an increased faecal isolation rate of the Gram-negative micro-organism Klebsiella pneumoniae has been reported from such patients. ${ }^{1}$

Radioimmunoassay with bovine vitreous humour and rabbit antivitreous serum has been used to determine immunological cross-reactivity with bacterial and mammalian tissue antigens. Klebsiella ultrasonicate preparation at a dose of $10 \mathrm{mg} / \mathrm{ml}$ was found to inhibit the binding of vitreous humour of $25-100 \%(p<0.001)$. Equivalent amounts of Streptococcus pyogenes, bovine haemoglobin, and

Table 5 Analysis of results on the 79 patients with $R A$

\begin{tabular}{|c|c|c|c|c|c|c|c|c|}
\hline & \multirow{2}{*}{$\begin{array}{l}\text { Upper limits } \\
\text { of } \\
\text { normal }\end{array}$} & \multicolumn{3}{|c|}{ Number of sera } & \multicolumn{4}{|c|}{ Kendal rank correlation coefficient } \\
\hline & & Elevated & Normal & $\boldsymbol{H A}$ & $H B$ & IF & Cart. & Nat. II \\
\hline $\begin{array}{l}\text { Den. II } \\
\text { Nat. II } \\
\text { Cart. } \\
\text { IF } \\
\text { H.B. } \\
\text { H.A. }\end{array}$ & $\begin{array}{l}<1300(\mu \mathrm{g} / 1) \\
<234(\mu \mathrm{g} / 1) \\
<15.6(\%) \\
<8(\text { titre }) \\
<8 \text { (titre) } \\
<8 \text { (titre) }\end{array}$ & $\begin{array}{l}57 \\
44 \\
43 \\
35 \\
30 \\
40\end{array}$ & $\begin{array}{l}22 \\
35 \\
36 \\
44 \\
49 \\
39\end{array}$ & $\begin{array}{l}0 \cdot 36^{*} \\
0 \cdot 70^{*} \\
0 \cdot 68^{*} \\
0 \cdot 69^{*} \\
0 \cdot 78^{*} \\
-\end{array}$ & $\begin{array}{l}0 \cdot 36^{*} \\
0 \cdot 64^{*} \\
0 \cdot 60^{*} \\
0 \cdot 60^{*} \\
-\end{array}$ & $\begin{array}{l}0.45^{*} \\
0.64^{*} \\
0.63^{*} \\
- \\
-\end{array}$ & $\begin{array}{l}0 \cdot 38^{*} \\
0 \cdot 70^{*} \\
- \\
- \\
-\end{array}$ & $\begin{array}{l}0.48^{*} \\
- \\
- \\
-\end{array}$ \\
\hline
\end{tabular}

$* \mathrm{p}<0.001$ 
hyaluronic acid had no inhibitory effect, while horse spleen ferritin was found to inhibit vitrous humour binding between 0 and $10 \%$.

These results indicate that klebsiella microorganisms have antigens which partially resemble some eyeball components. It is suggested that acute anterior uveitus of ankylosing spondylitis may be produced by anti-Gram-negative bacterial antibodies binding to cross-reacting eye antigens.

\section{Reference}

1 Kuberski T T, et al. Arthritis Rheum 1981; 4 Suppl: s 78 (abstr. 123).

Antinuclear antibodies in primary biliary cirrhosis. R. M. Bernstein, M. E. Callender, J. M. Neuberger, C. C. Bunn, R. Williams, G. R. V. Hughes. Rheumatology Unit, Hammersmith Hospital, London W12, and Liver Unit, King's College Hospital, London SE5.

Scleroderma and the sicca syndrome occur in patients with primary biliary cirrhosis (PBC).The scleroderma is usually mild and often associated with other features of the CREST syndrome. Since an antibody reactive with the centromere region of chromosomes has been reported in association with CREST syndrome, we examined sera from 110 consecutive patients with PBC using indirect immunofluorescence on a substrate of HEp-2 cells. Anticentromere antibody was found in 10/20 $(50 \%)$ patients who had PBC with scleroderma and $0 / 90(0 \%)$ patients without scleroderma $(p<0.0001)$. A second nuclear staining pattern of multiple large dots without staining of mitotic figures was observed in sera from $10 / 32(31 \%)$ patients with sicca syndrome confirmed by an abnormal Schirmer's test and $4 / 78(5 \%)$ patents without sicca syndrome $(p<0.001)$. Neither pattern was observed with sera from 80 patients with chronic active hepatitis.

Precipitating antibodies were detached by counter immunoelectrophoresis using saline extracts of rabbit thymus and human spleen in a system revealing the identity or nonidentity of antibodies in adjacent wells. Antibodies to Sm and RNP were not observed. However, anti-Ro (SS-A) occurred in $5 / 110(4.5 \%)$ patients with PBC, and antibodies to both Ro and La (SS-B) were found in 2/80 (2.5\%) patients with chronic active hepatitis.

In patients with PBC a newly described nuclear staining pattern of multiple large dots appears to be associated with sicca syndrome. Anticentromere antibody is highly specific for patients with scleroderma.
Myelography: a case for audit. $O$. A. Thurtle, K. A. Cleur, M. I. D. Cawley. Department of Rheumatology, Southampton General Hospital.

The increased frequency of myelography since aqueous media and techniques (radiculography) became available stimulated a review of indications for and results of this investigation in our department. Fifty-four case records were available from a total of 55 myelograms during 1976-80 inclusive. Patients were divisible into 3 groups according to clinical features and indications for investigation.

Group $A$. Five patients with nerve root signs attributed to lesions rather than protruded intervertebral disc (PID), including spondylolisthesis, tuberculosis, Paget's disease, and neoplasia. Myelography aided management in 4.

Group B. Twenty-six patients with root signs attributed to PID. Thirteen myelograms showed definite PID or root compression, of which 6 were treated surgically. Four others showed minor abnormalities, 1 had lumbar stenosis, and 8 were normal.

Group $C$. Twenty-three patients with obscure chronic back and/or leg pain without root signs? Thirteen of these 'desperation myelograms' were normal. Nine showed PID or root compression One had lumbar stenosis. None had surgery.

Conclusions. (1) Myelography has proved valuable in many patients with persistent root compression signs from any cause, as a guide to possible surgery. (2) In the absence of root signs myelography sometimes revealed a possible cause for symptoms but did not alter management. No unexpected neoplastic lesions were found. The investigation is of dubious value in this group. (3) Morbidity was common, occurring in $1 / 3$ of patients, but usually very mild. However, 3 patients had temporarily distressing side effects.

An unusual presentation of polymyositis complicating rheumatoid arthritis: the 'rag doll' syndrome. M. J. Dodd, M. Thompson. Department of Rheumatology, Royal Victoria Infirmary, Newcastle upon Tyne.

Severe polymyositis complicating rheumatoid arthritis is uncommon. This is in contrast to the focal and nodular interstitial myositis which may be found in up to $80 \%$ of patients with rheumatoid arthritis.

Labbate and Ehrlich ${ }^{1}$ reported a case of polymyositis masquerading as muscular dystrophy complicating a long history of rheumatoid arthritis, emphasising the degree of weakness in their patient. 
We report a further 3 patients suffering from rheumatoid arthritis who developed severe polymyositis. The distribution of clinical involvement of muscles was unusual with myopathic facies, severe involvement of neck, proximal upper limb and trunk muscles, and severe myasthenic symptoms and signs. This particular presentation is referred to colloquially as the 'rag doll' appearance.

\section{Reference}

1 Labbate V A, Ehrlich G. J Bone Jt Surg 1976; 58: 571.

How long should we talk to patients? V. Wright, R. Hopkins, K. Burton. Leeds.

In an era of high-technology medicine communication with patients remains of the highest importance. It has been suggested that a long interview with patients is counterproductive. If a short interview suffices, this has profound implications in a hectic rheumatic outpatient clinic. To study this, 13 questions to which 173 rheumatic outpatients most frequently wished to know the answer were made the basis of a structured interview. Two lengths of interview were conducted in a controlled trial (short being of 2 minutes duration; long ranging from 4 to 11 minutes). Sixty patients were referred directly from their general practitioners, and not being in any of the remedial professions were randomly assigned to one of the 2 groups. They were tested by an independent observer immediately after interview and one month later at their return appointment, using a questionnaire that covered the 13 items of information given to all the patients.

The longer interview resulted in significantly more information being retained. Of individual questions only 3 scored significantly better with the longer interview: those about the reasons for $x$-rays immediately after interview, the role of heredity, and the place of rest at delayed recall.

The effect of ultrasound on acute inflammation in the rat. D. Goddard, S. Klevenhagen, H. L. F. Currey. The London Hospital Medical College, London E1 2AD.

Ultrasound is widely used in the treatment of traumatic and inflammatory lesions. This is based on the belief that it is anti-inflammatory and can reduce inflammatory oedema. However, reliable experimental and clinical evidence for this is lacking. We have therefore tested the influence of ultrasound on the inflammatory response to irritant sponges implanted subcutaneously in the rat. This inflammation is sensitive to anti-inflammatory drugs in a a dose independent manner. ${ }^{12}$

Polyurethane foam cubes $\left(1 \mathrm{~cm}^{3}\right)$ impregnated with dead Myobacterium tuberculosis were implanted subcutaneously at 4 separate dorsal sites in each animal. Ultrasound was administered daily under water as shown in Table 6 . Rats were killed on day +4 and the protein content of the fluid exudate measured as the dry weight gain in each sponge.

In a dose range below that which caused animals to lose weight we were unable to detect any reduction in dry weight gain (Table 6). This is in striking contrast to the action of anti-inflammatory drugs. We have thus failed to show any anti-inflammatory effect of ultrasound in this model.

1 Clark A K, Vernon-Roberts B, Currey H L F. Ann Rheum Dis 1975; 34 : 326.

2 Woodland J, Vernon-Roberts B, Swettenham K V, Currey H L F. Ann Rheum Dis 1977; 36: 160.
}

Table 6 Dry weight gain $(M G:$ mean $\pm S D)$ of sponges treated or sham treated with ultrasound (transducer applied over back right sponge)

\begin{tabular}{|c|c|c|c|c|c|c|c|c|c|}
\hline \multirow{3}{*}{$\begin{array}{l}\text { Dose + } \\
\text { Rx time }\end{array}$} & \multirow{3}{*}{$\begin{array}{l}\text { Animal } \\
\text { number }\end{array}$} & \multicolumn{4}{|l|}{ Treated } & \multicolumn{4}{|c|}{ Sham treated } \\
\hline & & \multicolumn{2}{|l|}{ Front } & \multicolumn{2}{|l|}{ Back } & \multicolumn{2}{|l|}{ Front } & \multicolumn{2}{|l|}{ Back } \\
\hline & & Right & Left & Right & Left & Right & Left & Right & left \\
\hline $\begin{array}{l}0.5 \text { watt } \\
\mathrm{cm}^{-2} \text { for } \\
2 \frac{1}{2} \mathrm{~min}\end{array}$ & $\begin{array}{l}7 \mathrm{Rx} \\
7 \text { sham }\end{array}$ & $47 \pm 6$ & $48 \pm 10$ & $52 \pm 9$ & $50 \pm 8$ & $48 \pm 9$ & $47 \pm 16$ & $59 \pm 8$ & $47 \pm 6$ \\
\hline $\begin{array}{l}1 \text { watt } \\
\mathrm{cm}^{-2} \text { for } \\
2 \frac{1}{2} \mathrm{~min}\end{array}$ & $\begin{array}{l}13 \mathrm{Rx} \\
12 \text { sham }\end{array}$ & $53 \pm 10$ & $54 \pm 16$ & $49 \pm 15$ & $43 \pm 13$ & $52 \pm 11$ & $60 \pm 16$ & $57 \pm 11$ & $47 \pm 11$ \\
\hline $\begin{array}{l}1 \text { watt } \\
\mathrm{cm}^{-2} \text { for } \\
5 \mathrm{~min}\end{array}$ & $\begin{array}{l}6 \mathbf{R x} \\
5 \text { sham }\end{array}$ & $67 \pm 11$ & $62 \pm 13$ & $60 \pm 14$ & $58 \pm 17$ & $56 \pm 7$ & $68 \pm 7$ & $57 \pm 13$ & $54 \pm 9$ \\
\hline $\begin{array}{l}2 \text { watts } \\
\mathrm{cm}^{-2} \text { for } \\
5 \mathrm{~min}\end{array}$ & $\begin{array}{l}5 \text { Rx } \\
5 \text { sham }\end{array}$ & $49 \pm 16$ & $55 \pm 14$ & $46 \pm 14$ & $51 \pm 10$ & $63 \pm 6$ & $60 \pm 12$ & $58 \pm 5$ & $50 \pm 3$ \\
\hline
\end{tabular}

$\mathbf{R x}=$ treatment 
Renal function after prolonged, Heavy consumption of aspirin for rheumatoid arthritis. M. S. Akyol, M. Thompson, D. N. S. Kerr. Royal Victoria Infirmary, University of Newcastle upon Tyne, NE1 7RU.

Heavy consumption of analgesic mixtures containing aspirin causes analgesic nephropathy, but several studies suggest that aspirin alone carries no such hazard. However, few very heavy consumers of aspirin have been studied by sensitive tests of glomerular and tubular function.

Sixteen patients with rheumatoid arthritis aged 34-71 (mean 51) had consumed 5-37 (mean 16) kg of aspirin over 4-40 (mean 16) years. None had taken analgesic mixtures and only one had received other known nephrotoxins (gold). Plasma creatinine was normal in all $\left(46-90\right.$, mean $74, \mu \mathrm{mol} / 1$. ${ }^{51} \mathrm{Cr}$ EDTA clearance was slightly depressed in 2 patients $\left(69,71 \mathrm{ml} / \mathrm{min} / 1 \cdot 73 / \mathrm{m}^{2}\right)$ but the mean for the group (112) was normal. Urinary concentrating power during the intranasal desmopressin test was slightly depressed in 3 patients $(558,658,680$ mosm $/ \mathrm{kg})$ but the mean for the whole group $(820$ mosm $\mathrm{kg})$ was well up the normal range. None had proteinuria, haematuria, or significant abnormalities on microscopy. 10/16 had an increased excretion of B-NAG.

These patients had well preserved renal function for their age and no clinical evidence of analgesic nephropathy. The raised B-NAG excretion is a nonspecific sign of tubular damage similar to the shedding of epithelial cells after aspirin ingestion; neither appears to predict chronic renal damage.

Conclusion. This study showed no evidence of permanent renal damage fromconsumption of aspirin alone in the doses usually employed to treat rheumatoid arthritis over an average period of 16 years.

Effect of intra-articular yttrium-90 $\left({ }^{90} \mathrm{Y}\right)$ on chronic pyrophosphate arthropathy (CPA) of the knee. M. Doherty, P. A. Dieppe. Department of Medicine, Bristol Royal Infirmary, Bristol BS2 8HW.

No definitive treatment is currently available for CPA. The way in which CPPD crystals cause arthritis is unclear, but part of the damage may result from the synovitis. We have therefore investigated the effects of medical synovectomy by intra-articular injection of ${ }^{90} \mathrm{Y}$.

Fifteen patients ( 13 women, 2 men; age 58-91, mean $71 \mathrm{yr}$ ) with bilateral and symmetrical knee involvement were assessed for 6 months after receiving $20 \mathrm{mg}$ triamcinolone hexacetonide (TH) $+5 \mathrm{mCi}$ of ${ }^{90} \mathrm{Y}$ into one knee and $20 \mathrm{mg}$ $\mathrm{TH}+$ saline into the other (control) knee. The side to receive ${ }^{90} \mathrm{Y}$ was randomly allocated and was patient and observer blind.

After 6 months a significant reduction in pain, inactivity stiffness, joint circumference, and tenderness were produced on the ${ }^{90} \mathrm{Y}$ side $(\mathrm{p}<0.01)$. A significant difference between range of movement on the 2 sides was also present after 6 months $(\mathrm{p}<0.01)$, partly due to disease progression on the control side. Patient and observer preference predicted the treatment side in all 15 cases. No significant difference was seen in joint deformity, instability, or SF analysis.

This study suggests that intra-articular ${ }^{90} \mathrm{Y}$ is of benefit in CPA. The predilection of this condition for the knees of elderly patients makes such treatment highly suitable. The observed disease progression in the control knee emphasises the need for effective treatment.

The effects of 19-nortestosterone treatment on clinical and laboratory parameters in patients with systemic lupus erythematosus. R. A. Hazelton, A. McCruden, W. H. Stimson, R. D. Sturrock. University Department of Medicine, Centre for Rheumatic Diseases, Glasgow Royal Infirmary, andô Department of Biochemistry, University of Strath clyde.

It has been reported that 19-nortestosterone (19-nor) favourably influences the course of the lupus diathesis in the NZB/NZW mouse without producing inappropriate androgenic phenomena. ${ }^{1}$ Ten patients $(8 \mathrm{~F}, 2 \mathrm{M})$ with a wide variety of clinical manifestations of SLE were entered into an open trial. Each patient received an average total dose of 800 mg 19-nor in divided monthly doses for a mean treatment period of 6 months. E tensive clinical and laboratory parameters of disease activity were documented before, during, and after the trial.

Among the 10 patients 5 showed no deterioration or improvement. Of those who had an exacerbation 3 were female and 2 were male. Of the laboratory variables, platelets were shown to rise significantly $(p<0.05)$. Assays of $T \gamma$ lymphocytes showed that they returned to normal within the first 3 months. $T \mu$ lymphocytes were largely unchanged. Functional T cell suppressor activity remained consistently low.

This study has shown that 19-nor can modify the immune system in SLE, but that this was not necessarily associated with lessening of disease activity.

\footnotetext{
Reference

1 Verheul H, Stimson W, den Hollander F, Schuurs A Prophylactic and therapeutic effects of nandrolone and its decanoate ester (Deca-Durabolin) in murine lupus. Int J Immunopharmacol 1980; 2: 230.
} 
Oral gold therapy in rheumatoid arthritis. B. P. Hunt, P. J. L. Holt. Rheumatism Research Centre, Manchester Royal Infirmary, Manchester M13.

Auranofin has been studied in incremental doses to determine dose range, efficacy, and safety in RA. The initial daily dose was $4 \mathrm{mg}$ increasing to $6 \mathrm{mg}$ and then $3 \mathrm{mg}$ at 4-monthly intervals depending on response.

Thirty-one patients with definite or classical RA of at least 6 months' duration have entered the trial; 21 have completed at least 6 months. 12/21 patients have so far responded clinically (visual analogue, grip strength, articular index, global assessment). Improvement occurred in 6 at $4 \mathrm{mg}$ and 6 at $6 \mathrm{mg}$; 4 have withdrawn because of lack of benefit ( 2 on $6 \mathrm{mg}, 2$ on $8 \mathrm{mg}$ ). There has been no response to date in 3 patients $(2$ on $6 \mathrm{mg}, 1$ on $8 \mathrm{mg}$ ).

There has been a definite improvement in laboratory parameters (ESR, CRP, immunoglobulins, rheumatoid factor) in 8 ( 5 on $4 \mathrm{mg}$ and 3 on $6 \mathrm{mg}$ ) and an equivocal response in a further 4 (on $6 \mathrm{mg}$ ) but no convincing change in the remainder. Onset of response was commonly delayed by some 4 months (range 2-5). Sequential radiological assessment is being evaluated.

Auranofin has been well tolerated-slight loosening of the motions has been present in most patients. Neutropenia, eosinophilia, lymphopenia $(\times 2)$, and stomatitis each occurred singly in 5 patients. Serum gold levels showed considerable interpatient variability (200-1000 ng/ml) and bore little relationship to clinical response.

These results suggest that auranofin is an effective treatment for RA and provide information for the optimum design of further trials.

Rest in hospital for rheumatoid arthritis (RA) -a clinical and iaboratory study. J. A. Hunter, M. E. Billingham, P. J. Rooney. Centre for Rheumatic Diseases and Gartnavel General Hospital, Glasgow, and I C I, Macclesfield.

Clinical studies of inpatient care in RA showed a significant advantage over outpatient management. ${ }^{1}$ We have compared clinical improvements during admission with changes in acute-phase reactants. Thirty-six patients with classical or definite RA were admitted because of a recent flare. They were confined to bed for 4 days, and then gradually mobilised so that by the end of 2 weeks they moved about at will. After one week significant improvements $(p<0.025)$ occurred in articular index, pain score, number of tender joints, grip strength (right hand), and patients' and doctor's assessments. These were maintained in the second week when grip strength (left hand) also improved. Significant falls $(\mathrm{p}<0.05)$ in C-reactive protein (CRP), haptoglobin, and $\alpha_{1}$ acid glycoprotein (AGP) were detected only after 2 weeks, and, although a significant fall in ESR was absent, changes in AGP and ESR were significantly correlated.

Patients with intial CRP levels $>20 \mu \mathrm{g} / \mathrm{ml}$ were divided into 2 groups: those with a fall of $\geqslant 25 \%$ and those with a fall of $<15 \%$ over 2 weeks. Comparison of initial clinical and laboratory parameters in these groups showed no significant differences. In 7 outpatients assessed in a similar manner to clinical or laboratory changes were demonstrated. We conclude that there is laboratory evidence for partial remission of RA when patients are admitted for rest. Some will show changes comparable to those induced by second-line drugs over longer periods. Studies of drug therapy should not include assessments during inpatient care.

\section{Reference \\ 1 Lee P, et al. QJM 1974; 43: 205.}

Cyclofenil-a treatment for scleroderma? T. Gibson, R. Grahame. Guy's Hospital, London SE1 9RT.

Cyclofenil is a diphenyletheylene and a weak oestrogen with some antioestrogenic properties. It has been shown to influence connective tissue metabolism and an uncontrolled study has suggested that it may be an effective treatment for scleroderma. $^{1}$

Eleven patients with scleroderma were admitted to a double-blind cross-over study of cyclofenil $200 \mathrm{mg}$ t.d.s. and matching placebo, each given for 4 months in a randomised schedule. Subjective assessments of skin tightness, joint pain and stiffness, dyspnoea, dysphagia, and muscle weakness, together with measurements of palm print, grip strength, mouthopening, in-vivo skin elasticity, and respiratory function tests were performed at regular intervals. Four patients were withdrawn during cyclofenil treatment because of hepatotoxicity and one patient died with renal failure. None of the variables measured showed a consistent effect in favour of cyclofenil, and the results did not support previous assertions of therapeutic efficacy. A larger, controlled study would be necessary to determine whether occasional patients may benefit from this treatment.

\section{Reference}

1 Herbai G, Blom B, Bostrom H. Acta Med Scand 1977; 201 : 203. 